\title{
REFERENCES
}

1. R. P. Gosselin, Some integral inequalities, Proc. Amer. Math. Soc. 13 (1962), 378-384.

2. J. L. Lions and J. Peetre, Propriêtes d'espaces d'interpolation, C. R. Acad. Sci. Paris 253 (1961), 1747-1749.

3. J. Peetre, Relations d'inclusion entre quelques espaces fonctionnels, Kungl. Fysiogr. Sällsk. i Lund Förh. 30 (1960), 47-50.

4. L. N. Slobodeckij, On the embedding of the space $W_{p}^{l_{1}} \cdots, l_{n}$ into the space $H_{p}^{l_{1}}, \cdots, l_{n}$ of $S . M$. Nikolskij, Uspehi. Mat. Nauk 14 (1960), 176-180. (Russian)

5. M. Taibleson, Thesis, University of Chicago, Chicago, Ill., 1962.

UNIVERSITY OF CONNECTICUT

\section{ON RAISING FLOWS AND MAPPINGS}

BY R. D. ANDERSON ${ }^{1}$

Communicated by E. E. Moise, October 18, 1962

It is assumed that all spaces with which we are concerned are separable metric. Let $(G, X)$ be a transformation group with $G=I, R$ where $I$ is the additive group of integers and $R$ the reals. $(I, X)$ is called a discrete flow and $(R, X)$ a continuous flow. The orbit $O_{x}$ of a point $x \in X$ under a flow $(G, X)$ is the set of all $g x$ for $g \in G$. A flow $(G, Y)$ is imbedded in a flow $\left(G, Y^{\prime}\right)$ if $Y \subset Y^{\prime}$ and $(G, Y)$ is $\left(G, Y^{\prime}\right)$ cut down to $Y$. We say that $(G, X)$ is raised to $(G, Y)$ provided there is a mapping $\phi$ of $Y$ onto $X$ such that for each $y \in Y$ and $g \in G$, $\phi g(y)=g \phi(y)$. In this paper we establish that any discrete flow can be raised to a discrete flow on a zero-dimensional space and any continuous flow to a continuous flow on a 1-dimensional space. We shall note that these newly produced flows can be considered as imbedded in a discrete flow on the disc on the one hand and in a continuous flow on the solid torus in Euclidean 3-space on the other. Thus all continuous flows on compact metric spaces can be produced from continuous flows on the solid torus. We include some remarks about minimal flows in $\$ 3$.

1. Discrete flows. Any homeomorphism $g$ of $X$ onto $X$ generates a discrete flow on $X$ and in turn any discrete flow is generated by the unit of the group $G$. Let $g_{x}$ denote the unit of the group or the generating homeomorphism.

We wish to establish

${ }^{1}$ Alfred P. Sloan Research Fellow. 
TheOREM IA. Any discrete flow can be raised to a discrete flow on a zero-dimensional space. ${ }^{2}$

Before proceeding to the proof of Theorem IA we introduce some notation and conventions. The idea of the proof is rather simple but the notation is somewhat messy.

Let $H=\left\{h_{j}\right\}_{j \geq 0}$ denote the set of closures of the elements of a countable basis of $X$. (For notational consistency we suppose $X$ is not a finite set. The theorem is obvious for $X$ finite anyway.)

Let $N$ be a countably infinite collection of monotonic doubleended sequences $\left\{n_{i}(j)\right\}_{|i|<\infty}, 0 \leqq j<\infty$, of integers such that every integer is a term of some sequence of $N$ and for $j_{1} \neq j_{2}$ or $i_{1} \neq i_{2}$, $n_{i_{1}}\left(j_{1}\right) \neq n_{i_{2}}\left(j_{2}\right) . H$ and $N$ are in 1-1 correspondence given by $j$.

We define the $N$-shift of the set of all integers as that transformation which corresponds each integer to its successor in the particular element of $N$ of which it is a term. Under the $N$-shift each integer has a unique successor and a unique predecessor.

The homeomorphism $\sigma$ of the Cantor-Set. We let the CantorSet, $C$, be represented by the set of all double-ended sequences of zeros and ones with a specified binary (starting) point. To realize $C$ from the set of all ordinary sequences of zeros and ones, we need merely reinterpret the set of even numbers as the set of positive integers and the set of odd numbers as the set of negative integers.

We denote a point of $C$ by $p=\left\{p_{i}\right\} \quad(i<\infty, i>-\infty)$ where for each $i, p_{i}=0,1$ and $p_{0}$ (or the subscript 0 ) determines the binary point.

The homeomorphism $\sigma$ of $C$ onto itself is that induced by the $N$ shift on the set of subscripts of the $\left\{p_{i}\right\}$ representation of the points of $C$; in other words, $\sigma\left(\left\{p_{i}\right\}\right)=\left\{p_{i^{\prime}}\right\}$ where, for each $i$, the integer $i^{\prime}$ is the successor of $i$ and $p_{i^{\prime}}=0$ iff $p_{i}=0$. If $N$ had only one element, $\sigma$ would be the ordinary shift homeomorphism of $C$ onto itself.

It is clear that $\sigma$ is continuous and 1-1 onto.

Proof of Theorem IA. We shall exhibit a zero-dimensional space $Y$, a homeomorphism $g_{y}$ of $Y$ onto itself and a mapping $\phi$ of $Y$ onto $X$ such that $\phi g_{y}=g_{x} \phi$.

The space $Y$ is to be a subset of $X \times C$.

Let $\Phi$ denote the projection of $X \times C$ onto $X$.

Let $\bar{g}$ be the homeomorphism of $X \times C$ onto itself which is defined by

$$
\bar{g}[(x, p)]=(g(x), \sigma(p)) .
$$

Since both $\mathrm{g}$ and $\sigma$ are homeomorphisms, so is $\bar{g}$.

2 It has been pointed out to me by de Groot that Theorems IA and IIA can also be proved rather easily for completely regular spaces by use of his theorem on linearization of homeomorphisms and mappings. 
Let $Y$ be the set of all points $\left(x,\left\{p_{i}\right\}\right)$ of $X \times C$ except for those for which either

(a) for some $i$ and some $j, x \in g^{i}\left(h_{j}\right)$ and $p_{n_{i}}(j)=1$, or

(b) for some $i$ and some $j, x \notin \mathrm{Cl}\left(X \backslash g^{i}\left(h_{j}\right)\right)$ and $p_{n_{i}}(j)=0$.

The desired homeomorphism $g_{y}$ of $Y$ onto $Y$ is simply $\bar{g} \mid Y$. The mapping $\phi$ is $\Phi \mid Y$.

In the following remarks we show that $Y, g_{y}$, and $\phi$ are as needed for the theorem.

(1) $\Phi(Y)=X$ so that $\phi$ is onto. For any $x \in X$ and any index $k$, the criteria (a) and (b) guarantee that at most one of the two possible values of $p_{k}$ is excluded.

(2) $\phi$ is continuous for $\Phi$ is continuous.

(3) $Y$ is closed. By indexing by the sum of $|i|$ and $j, Y$ can be observed to be the intersection of a monotonic decreasing sequence of closed sets.

(4) The homeomorphism $\bar{g}$ carries $Y$ onto itself or, equivalently, $g_{Y}$ is a homeomorphism of $Y$ onto $Y$.

It suffices to observe that for any $\left(x,\left\{p_{i}\right\}\right)$, and $\left(g(x), \sigma\left(\left\{p_{i}\right\}\right)\right)$ either both are in $Y$ or neither is.

If $\left(x,\left\{p_{i}\right\}\right)$ fails to be in $Y$ because of condition (a) then, for some $i$ and some $j$, both

$$
x \in g^{i}\left(h_{j}\right) \quad \text { and } \quad p_{n_{i}}(j)=1 .
$$

But then applying $\bar{g}$ to $\left(x,\left\{p_{i}\right\}\right)$ we get $g(x) \notin g\left(g^{i}\left(h_{j}\right)\right)=g^{i+1}\left(h_{j}\right)$ and $p_{n_{i+1}}(j)=1$ which means that $\left(g(x), \sigma\left(\left\{p_{i}\right\}\right)\right)$ is not in $Y$.

Similar considerations suffice both for condition (b) and for the other order of implication. Thus $g_{Y}$ is a homeomorphism of $Y$ onto $Y$.

(5) $\phi g_{y}=g_{x} \phi$ since $\Phi \bar{g}=g_{x} \Phi$ by definition of $\Phi$ and $\bar{g}$ on $X \times C$.

(6) $Y$ is zero-dimensional.

We consider the Cantor-Set as a vertical middle-third set. Then $X \times C$ (and hence $Y$ ) is cut up into arbitrarily thin horizontal slices open and closed in $X \times C$. It suffices to observe that for any $\left(x,\left\{p_{i}\right\}\right)$ in $Y$ we can locate it in such a thin horizontal slice containing no point of $Y$ far away from it. But we shall see that the criterion (a) for a point not being in $Y$ implies that there is such a slice. If $h_{j}$ contains $x$ in its interior, if $h_{j}$ is small in $X$, and if $\left(x,\left\{p_{i}\right\}\right) \in Y$, then $p_{0}(j) \neq 0$ by condition (b) and thus $p_{0}(j)$ must be 1 . But for any $x^{\prime} \notin h_{j}$, every point of the form $\left(x^{\prime},\left\{p_{i}\right\}\right)$ with $\bar{p}_{0}(j)=1$ is not in $Y$ by condition (a). This implies that a sufficiently thin slice containing $\left(x,\left\{p_{i}\right\}\right)$, (defined by specifying finitely many of the $p_{i}$ including $\left.p_{0}(j)=1\right)$ contains no point far from $\left(x,\left\{p_{i}\right\}\right)$.

Thus Theorem IA is proved.

THEOREM IB. Any discrete flow on a compact metric space can be raised to a discrete flow on a Cantor-Set. 
Proof. The proof of Theorem IA with the observation (3) that the set $Y$ of the proof is closed asserts that the flow can be raised to a compact zero-dimensional space $Y^{\prime}$. Let $Y^{\prime} \times C$ be the desired Cantor-Set where $\phi$ is the projection from $Y^{\prime} \times C$ to $Y^{\prime}$ to $X$ and the homeomorphism on $Y^{\prime} \times C$ is the coordinate homeomorphism using the identity on $C$.

TheOREM IC. Any discrete flow on a compactum can be raised to a discrete flow imbedded in a flow, on a 2-dimensional disc, which is the identity on the boundary of the disc.

PRoof. This theorem follows immediately from Theorem IB and the known fact (see [5] for example) that for $C$ the Cantor-Set and $D$ the disc with $C \subset$ Int $D$, any homeomorphism of $C$ onto itself can be extended to a homeomorphism of $D$ onto itself holding the boundary pointwise fixed.

In [5] the condition on the boundary is not explicitly given but is inherent in the procedure.

By a mapping is meant a continuous transformation.

THEOREM IIA. Let $f_{x}$ be a mapping of $X$ onto itself. Then there exist a zero-dimensional space $Y$, a mapping $f_{\nu}$ of $Y$ onto $Y$ and a mapping $\phi$ of $Y$ onto $X$ such that $\phi f_{y}=f_{x} \phi$.

Proof. The proof of this theorem follows the proof of Theorem IA except that $f_{x}$ and $f_{y}$ are mappings and not homeomorphisms. The images of elements of $H$ under $f_{x}^{i}$ for $i<0$ are counter-images and can be used.

THEOREM IIB. Under the conditions of Theorem IIA, if $X$ is compact, then $Y$ can be taken to be the Cantor-Set.

2. Continuous flows. We assume $(G, X)$ a continuous flow on $X$. For any $g \in G$, we denote by $I(g)$ the additive group of iterates of $g$. Thus $(I(g), X)$ is the discrete flow generated by $g$.

Let $\mu$ be the imbedding of $(I(g), X)$ in $(G, X)$, induced by the identity injection of $I(g)$ in $G$.

Let $C(X)=X \times[0,1]$ be called the cylinder over $X$. Let $T C(X, g)$ denote the twisted cylinder over $X$ with respect to $g$, i.e., the image of $C(X)$ under a map $\theta$ which is $1-1$ except on $(X \times 0) \cup(X \times 1)$ where it is $2-1$ identifying $(x, 0)$ with $\left(g^{-1}(x), 1\right)$ for all $x \in X$.

We call $\theta(X \times 0)$ the base of the twisted cylinder. Let $R(g)$ denote the additive group of reals with $g$ as the unit. Then there exists a canonical continuous flow on $T C(X, g)$ denoted by $(R(g), T C(X, g))$ and parametrized by $[0,1]$ on $C(X) .^{3}$

${ }^{3}$ This method of constructing a continuous flow from a discrete flow is described in [2]. 
With the base of $T C(X, g)$ identified as $X$, we may consider $(R(g), T C(X, g))$ as a natural extension of $(I(g), X)$ or conversely $(I(g), X)$ as naturally imbedded in $(R(g), T C(X, g))$. The orbit which contains a point $\theta(x, 0)$ of the base of the twisted cylinder is the image under $\theta$ of the union of the vertical intervals in $C(x)$ over the orbit of $(x, 0)$ under $I(g)$ acting on $(X \times 0)$.

If $\phi$ is the mapping (of the proof of Theorem IA) of $Y$ onto $X$ and $i_{y}$ and $i_{x}$ are the natural imbeddings of $(I(g), Y)$ and $(I(g), X)$ in $(R(g), T C(Y, g))$ and $(R(g), T C(X, g))$ respectively, then $i_{\nu}, i_{x}$, and $\phi$ induce a mapping $\phi^{\prime}$ of $(T C(Y, g))$ onto $T C(X, g)$ such that for any $\tilde{g} \in R(g), \tilde{g} \phi^{\prime}=\phi^{\prime} \tilde{g}$. Let $\eta$ be the mapping of $T C(X, g)$ onto $X$ satisfying the relationship $\eta i_{x}=\mu$ and such that the composition of $\phi^{\prime}$ followed by $\eta$ is a mapping of $T C(Y, g)$ onto $X$ which commutes with every $g$ in $R(g)$. But $Y$ is zero-dimensional in Theorem IA and thus $T C(Y, g)$ is one-dimensional (for locally it is the product of $Y$ by $[0,1])$. If the original space $X$ is compact then all the derived spaces of this development also are compact. Thus we have established the theorems:

THEOREM IIIA. Any continuous flow can be raised to a continuous flow on a 1-dimensional space.

THEOREM IIIB. Any continuous flow on a compactum can be raised to a continuous flow on a 1-dimensional compactum.

Finally we note that if we use the twisted cylinder over the discrete flow on the disc of Theorem IC, the twisted cylinder itself is the solid torus-for our flow was the identity on the boundary of the disc. Thus the argument of this section establishes

ThEOREM IIIC. Any continuous flow on a compactum can be raised to a continuous flow imbedded in a flow on a solid torus.

3. Minimality. A flow $(G, X)$ (discrete or continuous) is called minimal if the closure of each orbit is $X$. $p$-adic flows on the CantorSet and irrational rotations on tori (discrete or continuous) are examples of minimal flows.

REMARK. Since the image of a dense set under an onto map is dense, it is immediate that if $(G, Y)$ and $(G, X)$ are flows, $\phi$ maps $Y$ onto $X, \phi$ commutes with each element of $G$, and $(G, Y)$ is minimal, then $(G, X)$ is minimal.

TheOREM IV. If $X$ is compact and $(G, X)$ is minimal, then it can be prescribed in Theorems IB and IIIB that $(G, Y)$ is minimal.

Proof. Given any flow on a compact space, $Y$, there exists a compact subspace $Y^{\prime}$ on which the flow, cut down to $Y^{\prime}$, is minimal, see 
[2] or [4] for example. But if the image of $Y^{\prime}$ under the projection is a proper subset $X^{\prime}$ of $X$, then for any $x^{\prime} \in X^{\prime}, \theta\left(x^{\prime}\right)$ is not dense in $X$, contradicting our hypothesis. Thus $(G, X)$ is raised to $\left(G, Y^{\prime}\right)$. If $X$ were finite, then $Y^{\prime}$ of our construction might be finite but we may raise $(G, X)$ to a minimal $p$-adic flow on $C$ in such case anyway.

Theorem V. Any continuous minimal flow on a compactum can be raised to a flow on an indecomposable continuum every composant of which is a continuous 1-1 image of the line.

Proof. It is known (and follows from a Baire category type argument) that if $(G, X)$ is the continuous minimal flow of the hypothesis, then for some $g \in G$, the induced $(I(g), X)$ is also a minimal flow. But from Theorem IV, $(I(g), X)$ can be raised to a minimal discrete flow on a Cantor-Set. Now employing the proof of Theorem IIIB we note that the twisted cylinder of $\$ 2$ is just such an indecomposable continuum.

\section{REFERENCES}

1. V. V. Nemytski and V. V. Stepanov, Qualitative theory of differential equations, English Transl., Princeton Univ. Press, Princeton, N. J., 1960; Moscow-Leningrad 2nd ed., 1949.

2. V. V. Nemytski, Topological problems of the theory of dynamical systems, Amer. Math. Soc. Transl. (103); Uspehi Mat. Nauk 4 (1949) no. 6 (34), 91-153.

3. W. H. Gottschalk and G. A. Hedlund, Topological dynamics, Amer. Math. Soc. Colloq. Publ. Vol. 36, Amer. Math. Soc., Providence, R. I., 1955.

4. W. H. Gottschalk, Minimal sets: An introduction to topological dynamics, Bull. Amer. Math. Soc. 64 (1958), 336-351.

5. C. Kuratowski, Topologie. II, Warsaw, 1952.

Louisiana State University and Mathematisch Centrum, Amsterdam 